\title{
ON GAP SERIES AND \\ THE LEHTO-VIRTANEN MAXIMUM PRINCIPLE
}

CH. POMMERENKE*

\section{Hadamard gap series}

Let $\boldsymbol{D}$ denote the unit disk. We consider first functions

$$
f(z)=\sum_{k=0}^{\infty} a_{k} z^{n_{k}} \quad(z \in \boldsymbol{D})
$$

with Hadamard gaps, i.e.,

$$
\frac{n_{k+1}}{n_{k}} \geqq \lambda>1 \quad \text { for } \quad k=1,2, \ldots
$$

We assume that the coefficients are unbounded. Then the maximal term satisfies

$$
\mu(r) \equiv \max _{k}\left|a_{k}\right| r^{n_{k}} \rightarrow \infty \quad \text { as } \quad r \rightarrow 1-0 .
$$

T. Murai [6] has shown that $f$ has the asymptotic value $\infty$ at every point of $\partial D$. This problem had been raised and partially answered by G. R. MacLane [5, p. 46]; compare also [2]. A quantitative version of Murai's result was recently proved by D. Gnuschke and the author:

Theorem 1 [3]. Let $f$ have Hadamard gaps and unbounded coefficients. Then, for every $\zeta \in \partial D$, there is a Jordan arc $C$ ending at $\zeta$ such that

$$
\frac{|f(z)|}{\mu(|z|)}>\alpha \quad \text { for } \quad z \in C
$$

where $\alpha$ is a positive constant depending only on $\lambda$.

The proof uses the Lehto-Virtanen maximum principle [4]. We shall prove a variant of this principle and deduce a partial converse of Theorem 1:

Theorem 2. Let $f$ have Hadamard gaps and let

$$
\mu\left(\frac{1+r}{2}\right) \leqq M_{1} \mu(r) \quad\left(\frac{1}{2} \leqq r<1\right), \quad \mu(r) \rightarrow \infty \quad(r \rightarrow 1-0) .
$$

*) This research was in part supported by the British SERC. 
Then there is a constant $M_{2}$ such that, for almost all $\zeta \in \partial D$,

$$
\inf _{z \in C} \frac{|f(z)|}{\mu(|z|)} \leqq M_{2}
$$

for every Jordan arc $C$ ending at $\zeta$.

It follows that Theorem 1 is best possible for every Hadamard function $f$ satisfying (1.5). Note that this refers only to the approach along an arc. The average size of $|f(z)| / \mu(r)$ on $|z|=r$ is much larger if

$$
\frac{1}{2 \pi} \int_{0}^{2 \pi}\left|\frac{f\left(r e^{i \theta}\right)}{\mu(r)}\right|^{2} d \theta=\sum_{k=0}^{\infty}\left(\frac{\left|a_{k}\right| r^{n_{k}}}{\mu(r)}\right)^{2} \rightarrow+\infty \quad \text { as } \quad r \rightarrow 1-0 .
$$

The central limit theorem for Hadamard gap series [8, p. 264] gives more precise information about the average size.

We use the assumption of lacunarity only in connection with the following lemma, a variant of a standard result [1, Lemma 2.1].

Lemma 1. If $f$ has Hadamard gaps and if

$$
\mu\left(\frac{1+r}{2}\right) \leqq M_{1} \mu(r) \quad \text { for } \quad \frac{1}{2} \leqq r<1
$$

for some constant $M_{1}$, then there exists $M$ such that

$$
\left(1-|z|^{2}\right)\left|f^{\prime}(z)\right| \leqq M \mu(|z|) \quad \text { for } \quad z \in D .
$$

Proof. Let $|z|=r \geqq 1 / 2$. Then, by (1.3) and (1.7),

$$
\left|a_{k}\right| r^{n_{k} / 2} \leqq \mu(\sqrt{r}) \leqq M_{1} \mu(r) \quad(k=0,1, \ldots) .
$$

Hence we see from (1.1) that

$$
r\left|f^{\prime}(z)\right| \leqq \sum_{k=0}^{\infty} n_{k}\left|a_{k}\right| r^{n_{k}} \leqq M_{1} \mu(r) \sum_{k=0}^{\infty} n_{k} r^{n_{k} / 2} .
$$

Using the fact that $n_{k} / n_{j} \leqq \lambda^{-(j-k)}$ for $k \leqq j$, we deduce that

$$
\begin{gathered}
\frac{r\left|f^{\prime}(z)\right|}{1-\sqrt{\bar{r}}} \leqq M_{1} \mu(r) \sum_{k=0}^{\infty}\left(\sum_{n_{k} \leqq m} n_{k}\right) r^{m / 2} \\
\leqq \frac{M_{1} \lambda \mu(r)}{\lambda-1} \sum_{k=0}^{\infty}(m+1) r^{m / 2}=\frac{M_{1} \lambda \mu(r)}{(\lambda-1)(1-\sqrt{r})^{2}},
\end{gathered}
$$

and this implies (1.8). 


\section{A Bloch-type condition}

We drop now the assumption that $f$ has Hadamard gaps and write

$$
f(z)=\sum_{n=0}^{\infty} a_{n} z^{n}, \quad \mu(r)=\max _{n}\left|a_{n}\right| r^{n} .
$$

We consider the condition

$$
\left(1-|z|^{2}\right)\left|f^{\prime}(z)\right| \leqq M \mu(|z|) \quad(z \in \boldsymbol{D})
$$

where $M$ is a constant. It holds for all Bloch functions and also for all Hadamard series satisfying (1.7) as Lemma 1 states.

Lemma 2. If (2.2) holds, then

$$
\left(1-r^{2}\right) \mu^{\prime}(r) \leqq M \mu(r) \quad(0 \leqq r<1)
$$

where $\mu^{\prime}$ denotes the right-hand derivative.

Proof. There exists $v$ such that $\mu(x)=\left|a_{v}\right| x^{v}$ in some interval to the right of $r$. Hence (2.2) shows that

$$
\mu^{\prime}(r)=v\left|a_{v}\right| r^{\nu-1} \leqq \max _{|z|=r}\left|f^{\prime}(z)\right| \leqq \frac{M \mu(r)}{1-r^{2}} .
$$

Remarks. 1. It follows from Lemma 2 that, conversely, condition (1.7) is a consequence of (1.8).

2. It is not difficult to show that

$$
\mu(r) \leqq \frac{e}{2} \max \left(1-|z|^{2}\right)\left|f^{\prime}(z)\right| \quad(0 \leqq r<1) .
$$

Hence the constant in (2.2) satisfies $M \geqq 2 e^{-1}$.

The next result is a variant of a result of $\mathrm{O}$. Lehto and K. I. Virtanen [4, Theorem 7]. For $\zeta \in \partial D$, we consider the Stolz angle

$$
\Delta_{\varrho}(\zeta)=\left\{|\arg (1-\bar{\zeta} z)|<\frac{\pi}{4}, \quad|z-\zeta|<\varrho\right\} \quad(\varrho>0)
$$

of opening $\pi / 2$ (which could be replaced by any number $<\pi$ ).

Theorem 3. Let $f$ be analytic in $D$ and let (2.2) be statisfied. Let, $C$ be a Jordan arc ending at $\zeta \in \partial D$. If

$$
|f(z)| \geqq M_{2} \mu(|z|) \text { for } z \in C
$$

where $M_{2}$ is a constant depending only on $M$, then

$$
|f(z)| \geqq \mu(|z|) \text { for } \quad z \in \Delta_{\varrho}(\zeta)
$$

if $\varrho$ is sufficiently small. 
Proof of Theorem 2. Let $E$ be the set of all $\zeta \in \partial D$ such that (2.6) holds for some curve $C$ ending at $\zeta$. It follows from (1.5) and Lemma 1 that (2.2) is satisfied. Hence Theorem 3 shows that (2.7) holds for $\zeta \in E, \varrho=\varrho(\zeta)>0$.

Since $\mu(r) \rightarrow \infty$ as $r \rightarrow 1-0$, we deduce from (2.7) that $f(z) \rightarrow \infty$ as $z \rightarrow \zeta$ in a Stolz angle at $\zeta \in E$. Hence it follows from Plessner's theorem (e.g. [7, p. 324]) that mes $E=0$. This proves Theorem 2 because (1.6) holds for every $\zeta \in \partial D \backslash E$.

In order to prove Theorem 3 we introduce

$$
\psi(z)=\frac{\mu(|z|)}{|f(z)|} \quad(z \in \boldsymbol{D})
$$

The function (see (2.1))

$$
\log \psi(z)=\max _{n}\left(\log \left|a_{n}\right|+n \log |z|\right)-\log |f(z)|
$$

is subharmonic in $\boldsymbol{D}$ except for logarithmic poles at the zeros of $f$.

Furthermore, we see that, with $z=r e^{i \theta}$,

$$
\begin{gathered}
\left|\frac{\partial \psi}{\partial r}\right|=\left|\frac{\mu^{\prime}}{|f|}-\frac{\mu}{|f|} \operatorname{Re}\left[e^{i \theta} \frac{f^{\prime}}{f}\right]\right| \leqq \frac{\mu^{\prime}}{|f|}+\frac{\mu\left|f^{\prime}\right|}{|f|^{2}}, \\
\left|\frac{1}{r} \frac{\partial \psi}{\partial \theta}\right|=\frac{\mu}{|f|}\left|\operatorname{Re}\left[i e^{i \theta} \frac{f^{\prime}}{f}\right]\right| \leqq \frac{\mu\left|f^{\prime}\right|}{|f|^{2}},
\end{gathered}
$$

where $\mu^{\prime}$ denotes the right-hand derivative. Hence

$$
\frac{|\operatorname{grad} \psi|}{1+\psi^{2}} \leqq \frac{\mu^{\prime}|f|+2 \mu\left|f^{\prime}\right|}{\mu^{2}+|f|^{2}} \leqq \frac{\mu^{\prime}}{2 \mu}+\frac{2\left|f^{\prime}\right|}{\mu},
$$

and it follows from (2.2) and (2.3) that

$$
\frac{|\operatorname{grad} \psi(z)|}{1+\psi(z)^{2}} \leqq \frac{5}{2} \frac{M}{1-|z|^{2}} \text { for } \quad z \in D .
$$

Lemma 3. Let $0<\psi(z) \leqq+\infty(z \in D)$, let $\log \psi$ be subharmonic in $\boldsymbol{D}$ except for logarithmic poles and let (2.10) be satisfied. Let $G$ be a domain in $D$ bounded by an arc of $\partial D$ and a circular arc $A$ forming an angle $4 \pi / 5$ with $\partial D$. If

$$
\psi(z) \leqq e^{-K} \quad(z \in \partial G \backslash A), \quad K=2 \pi M / \sin \frac{\pi}{5}
$$

then $\psi(z) \leqq 1$ for $z \in G$.

This lemma is closely related to the Lehto-Virtanen maximum principle [4, Theorem 7] [7, Theorem 9.1]. We have specialized the parameters for an easier statement. Note that $\psi$ need not be the modulus of a meromorphic function. 
Proof (compare [7, pp. 264/265]). Suppose $\psi(z) \leqq 1 \quad(z \in G)$ does not hold. Then there exists a circular arc $B$ with the same endpoints as $A$ forming an angle $\beta<4 \pi / 5$ with $\partial D$ such that $\psi(z) \leqq 1$ in the part $H$ of $G \backslash B$ with $\partial H \subset \partial G \cup B$ and furthermore $\psi\left(z_{0}\right)=1$ for some $z_{0} \in B$. If $\gamma$ is a Möbius transformation of $\boldsymbol{D}$ onto $\boldsymbol{D}$ then $\psi \circ \gamma$ satisfies the same assumptions. Hence we may assume that $A$ (and thus $B$ ) ends at \pm 1 and that $z_{0}=i y_{0}$.

The subharmonic function

$$
u(z)=\log \psi(z)+\frac{K}{\beta}\left(\arg \frac{1+z}{1-z}-\frac{\pi}{2}\right) \quad(z \in H)
$$

is bounded by $-K$ both on $B \cap \partial H$ (because $\psi(z) \leqq 1$ ) and on $\partial G \backslash B$ (because of (2.11)). Hence the maximum principle shows that $u(z) \leqq-K$ for $z \in H$.

We conclude that

$$
K+\frac{K}{\beta}\left(2 \arctan y-\frac{\pi}{2}\right) \leqq-\log \psi(z) \quad(i y \in H) .
$$

Since both sides vanish for $y=y_{0}$ we see that

$$
\frac{2 K}{\beta\left(1+y_{0}^{2}\right)} \leqq-\left.\frac{\partial}{\partial y} \log \psi(i y)\right|_{y=y_{0}} \leqq \frac{5 M}{1-y_{0}^{2}},
$$

by $(2.10)$ and because $\psi\left(i y_{0}\right)=1$. By (2.11), this is equivalent to $\sin \beta / \beta \leqq$ $(5 / 4 \pi) \sin (4 \pi / 5)$, and this contradicts $\beta<4 \pi / 5$.

Proof of Theorem 3. We have seen that the function $\psi$ defined by (2.8) satisfies the assumptions of Lemma 3. Let $C^{\prime} \subset D$ be the subarc of $C$ obtained by deleting a small part near $\partial D$. Let $A$ be the circular arc through the endpoints of $C^{\prime}$ that forms the angle $4 \pi / 5$ with $\partial D$ and let $G^{\prime}$ be the domain between $C^{\prime}$ and $A$; if $G$ is not connected, we apply the argument to the components.

We choose $M_{2}=\exp [-2 \pi M / \sin (\pi / 5)]$. If (2.6) holds, then $\psi(z) \leqq 1 / M_{2}$ for $z \in C$. Hence (2.11) is satisfied and we conclude that $\psi(z) \leqq 1$ for $z \in G^{\prime}$. Letting $C^{\prime} \rightarrow C$ we see that $\psi(z) \leqq 1$ in the corresponding domain $G$. We apply the same argument to the arc $A^{*}$ with the angle $4 \pi / 5$ in the opposite direction and obtain $\psi(z) \leqq 1$ for $z \in G^{*}$. This proves $(2.7)$ because $\Delta_{\varrho}(\zeta) \subset G \cup G^{*}$ if $\varrho$ is sufficiently small.

I want to thank Professor W. K. Hayman for our discussions about this problem.

\section{References}

[1] Anderson, J. M., J. Clunie, and Ch. Pommerenke: On Bloch functions and normal functions. J. Reine Angew. Math. 270, 1974, 12-37.

[2] BinMore, K. G., and R. Hornblower: Boundary behaviour of functions with Hadamard gaps. - Nagoya Math. J. 48, 1972, 173-181.

[3] Gnuschke, D., and CH. Pommerenke: On the growth of functions with Hadamard gaps. - J. London Math. Soc. (to appear). 
[4] Lehto, O., and K. I. ViRTANEN: Boundary behaviour and normal meromorphic functions. Acta Math. 97, 1957, 47-65.

[5] MacLane, G. R.: Asymptotic values of holomorphic functions. - Rice Univ. Studies 49 no. 1, $1963,1-83$.

[6] MURAI, T.: The boundary behaviour of Hadamard lacunary series. - Nagoya Math. J. 89, 1983, 65-76.

[7] Pommerenke, Сн.: Univalent functions. - Vandenhoeck \& Ruprecht, Göttingen, 1975.

[8] Zygmund, A.: Trigonometric series. Vol. II. - 2nd edition. Cambridge University Press, Cambridge, 1968.

Technische Universität Berlin

Fachbereich Mathematik

D 1000 Berlin 12

Bundesrepublik Deutschland

Received 8 November 1983 